\section{Recomendaciones para la realización de intervenciones pulmonares durante la pandemia COVID-19}

\author{
SEBASTIÁN FERNÁNDEZ-BUSSY ${ }^{1}$, OLIVIA SÁNCHEZ², \\ MIHIR PARIKH ${ }^{3}$, ALICHIA PATON ${ }^{3}$, FAYEZ KHEIR ${ }^{3}$, \\ ALEX CHEE $^{3}$, RACHEL MARTÍNEZ ${ }^{3}$, SEBASTIÁN GANDO ${ }^{4}$, \\ MARIO LANZA ${ }^{5}$, ALEJANDRA CANIAS ${ }^{6}$, \\ GONZALO LABARCA ${ }^{7}$, MARCO ANTONIO GARCIA ${ }^{8}$, \\ ROBERTO DURE ${ }^{9}$, DAVID ABIA-TRUJILLO ${ }^{1}$, \\ ARTEMIO OSCAR GARCÍA ${ }^{10}$, PABLO RUBINSTEIN ${ }^{11}$, \\ HUGO DE OLIVEIRA ${ }^{12}$, JAVIER FLANDES ${ }^{13}$, \\ ERIK FOLCH ${ }^{14}$, ADNAN MAJID $^{3}$
}

\section{Recommendations for performing interventions during the COVID-19 pandemic}

Coronavirus infection (SARS-CoV-2), is a pandemic disease declared by the World Health Organization (WHO). This disease reports a high risk of contagion, especially by the transmission of aerosols in health care workers. In this scenario, aerosol exposure is increased in various procedures related to the airway, lungs, and pleural space. For this reason, it is important to have recommendations that reduce the risk of exposure and infection with COVID-19. In this document, a team of international specialists in interventional pulmonology elaborated a series of recommendations, based on the available evidence to define the risk stratification, diagnostic methods and technical considerations on procedures such as bronchoscopy, tracheostomy, and pleural procedures among others. As well as the precautions to reduce the risk of contagion when carrying out pulmonary interventions.

(Rev Med Chile 2020; 148: 689-696)

Key words: Coronavirus; COVID-19; tracheostomy; Bronchoscopy; Interventional pulmonology.

\author{
'Division of Pulmonary and Critical Care. \\ Mayo Clinic Florida. USA. \\ ${ }^{2}$ Instituto Enfermedades Respiratorias (INER) \\ Universidad Autónoma de México. México. \\ ${ }^{3}$ Division of Thoracic Surgery and \\ Interventional Pulmonology. Beth Israel \\ Deaconess Medical Center-Harvard Medical \\ School. USA \\ ${ }^{4}$ Sanatorio Las Lomas de San Isidro. Buenos \\ Aires, Argentina. \\ ${ }^{5}$ Hospital Honduras Medical Center. \\ Tegucigalpa, Honduras. \\ ${ }^{6}$ Pontificia Universidad Javeriana. Hospital \\ Universitario San Ignacio. Bogotá, Colombia. \\ ${ }^{7}$ Facultad de Medicina, Universidad de \\ Concepción, Chile. \\ ${ }^{8}$ Pulmonary Medicine. Instituto Nacional de \\ Tórax. La Paz, Bolivia. \\ ${ }^{9}$ Hospital Muñiz. Buenos Aires. Argentina. \\ ${ }^{10} \mathrm{Hospital}$ Posadas y Hospital Británico. \\ Buenos Aires, Argentina. \\ ${ }^{11}$ Hospital Universitari General de Catalunya. \\ Barcelona. España. \\ ${ }^{12}$ Hospital de Clínicas de Porto Alegre. \\ Universidade Federal do Rio Grande do Sul, \\ Brasil. \\ ${ }^{13}$ Unidad de Broncoscopias y Neumología \\ Intervencionista. Hospital Universitario \\ Fundación Jiménez Díaz. IIS-FJD CIBERES. \\ Madrid, España. \\ ${ }^{14}$ Division of Pulmonary and Critical Care \\ Medicine. Massachusetts General Hospital. \\ Harvard Medical School. USA. \\ Financiamiento: sin financiamiento externo \\ Conflicto de interés: Los autores no reportan \\ conflicto de interés. \\ Recibido el 8 de abril de 2020, aceptado el 4 \\ de mayo de 2020. \\ Autor de Correspondencia \\ Dr. Sebastián Fernández-Bussy \\ Division of Pulmonary and Critical Care. \\ Mayo Clinic Florida. USA \\ Fernandez-Bussy.Sebastian@mayo.edu
}

E l coronavirus (SARS-CoV-2), es un agente etiológico causante de neumonía viral emergente, comúnmente llamado COVID-19. Este microorganismo produce cuadros de neumonía asociado a falla ventilatoria, principalmente por el desarrollo de distrés respiratorio agudo. Dentro de las características de este virus, se encuentra la alta capacidad de contagio, lo que se asocia a una tasa de infecciones asociadas a la atención en salud con un rango de 20\%-30\%.

En este escenario, los procedimientos asociados a intervenciones de la vía aérea poseen un riesgo aumentado de contagio y de exposición del personal de salud por la generación de ae- 
rosoles respiratorios. Es importante establecer que procedimientos son electivos, prioritarios y urgentes. Esto reducirá el riesgo de contagio en el personal, así como el aumento de nuevos casos de COVID-19.

El objetivo de este documento es entregar recomendaciones para disminuir el contagio de SARS-CoV-2 en relación a procedimientos que impliquen intervenciones pulmonares.

\section{Objetivos}

- Reducir el posible contagio de la infección por COVID-19 en pacientes, acompañantes y personal de salud.

- Detección temprana de los pacientes asintomáticos y sintomáticos en el triaje, que hayan estado en riesgo de contagio por COVID-19.

- Establecer un plan de trabajo en pacientes con sospecha o confirmación de infección por COVID-19 previo a la realización de procedimientos de intervencionismo pulmonar.

- Establecer un plan de trabajo en pacientes sin sospecha de infección por COVID-19 y sin necesidad de intervención pulmonar urgente (no necesario para cambio de conducta inmediata).

\section{Metodología}

Se contactaron especialistas broncopulmonares de distintos centros de Norteamérica, Centro América, Sudamérica y España. Cada participante fue contactado mediante correo electrónico, con la finalidad de lograr consenso en las recomendaciones. De manera adicional, se identificó mediante base de datos PubMed (MEDLINE), referencias actualizadas usando los términos MeSH [SARS virus], [COVID-19], en combinación con [Bronchoscopy], [Tracheostomy], and [Diagnostic Techniques, Respiratory System]. Como criterio de inclusión se consideraron estudios clínicos y documentos de consenso y/o guías de práctica clínica publicadas por otras sociedades científicas, restringido al inglés. La última fecha de actualización de la búsqueda de referencias fue el 6 de abril de 2020, incluyendo un total de 5 artículos de referencia ${ }^{1-5}$.

\section{Recomendaciones respecto a estratificación de procedimientos pulmonares}

Con el objetivo de minimizar el riesgo de una posible infección por COVID-19, los procedimientos de intervención pulmonar (broncoscopia, pleurales o misceláneos) deben ser estratificados en electivos ( $\geq 4$ semanas), prioritarios ( $<4$ semanas) y urgentes $(<48 \mathrm{~h})$.

\section{A. Recomendaciones respecto a estratificación de broncoscopía}

\section{Procedimientos electivos}

a) Biopsias de nódulos pulmonares con bajo o moderado riesgo de invasión basado en la estratificación de riesgo de malignidad (vidrio esmerilado, sólido, mixtos o lesiones $<2 \mathrm{~cm}$ ).

b) Tos crónica (sin imagen sugerente de etiología) o hemoptisis no masiva en presencia de una tomografía normal o bronquiectasia.

c) Lavado bronco-alveolar (LBA) en pacientes con sospecha de micobacterias atípicas (MAC) con mínimos síntomas.

d) Sospecha de sarcoidosis sin síntomas.

e) Válvulas endobronquiales en pacientes con enfisema severo.

f) Termoplastía bronquial para asma severa.

g) Criobiopsias transbronquiales en enfermedad pulmonar intersticial difusa (EPID) estable.

h) Exploración de pacientes con colapso dinámico de la vía aérea.

i) Broncoscopia rígida para colocación de stents en traqueomalacia sin falla respiratoria.

\section{Procedimientos Prioritarios:}

a) Biopsia o estadificación de nódulos de alto riesgo

i. $\geq 2 \mathrm{~cm}$, lesiones centrales, $\mathrm{o}$ asociado a adenopatías.

ii. Considerar biopsia percutánea para nódulos periféricos (1/3 del tórax) sin adenopatías.

iii. Considerar hacer positron emission tomography (PET) para estratificar antes de realizar broncoscopia.

b) Biopsia de pacientes con cáncer conocido con nuevas lesiones que modifiquen el tratamiento.

c) Sospecha de sarcoidosis con síntomas.

d) Sospecha de neumonitis inducida por drogas (COVID-19 negativos). 
e) LBA en paciente con sospecha de tuberculosis sin esputo espontaneo (previo a decidir realizar broncoscopia, se deben realizar todas las pruebas diagnósticas alternativas).

\section{Procedimientos urgentes:}

- Cuerpos extraños.

- Hemoptisis que ponga en riesgo la vida.

- Obstrucción de la vía aérea central. Lesiones de la vía aérea con atelectasias lobares.

- Fístulas (traqueo-esofágicas, broncopleurales, alveolos pleurales).

- Atelectasia pulmonar o lobar con hipoxemia en terapia intensiva que no responda a medidas de fisioterapia respiratoria.

- Nuevos infiltrados pulmonares en pacientes inmunocomprometidos (COVID-19 Negativos).

\section{B. Recomendaciones respecto a estratificación de procedimientos pleurales}

1. Procedimientos electivos:

a) Toracocentesis en pacientes con derrame pleural asintomático.

b) Catéteres pleurales tunelizados de derrames pleurales asintomáticos.

c) Toracoscopias médicas con toma de biopsia, pleurodesis y/o colocación de catéteres tunelizados en pacientes con baja o intermedia sospecha de malignidad.

2. Procedimientos prioritarios:

a) Toracocentesis en pacientes con derrame pleural sintomático.

b) Catéteres pleurales tunelizados en derrames pleurales sintomáticos.

c) Tubos torácicos en derrame pleural sintomático.

d) Toracoscopia médica con toma de biopsia pleural en pacientes con alta sospecha de tuberculosis o malignidad.

3. Procedimientos urgentes:

a) Tubos torácicos en pacientes con neumotórax a tensión, derrame pleural sintomático, hemotórax, derrame pleural paraneumónico complicado o empiema.
C. Recomendaciones respecto a estratificación de procedimientos misceláneos

1. Procedimientos electivos:

a) Traqueostomía en paciente estable.

2. Procedimientos prioritarios:

a) Traqueostomía en pacientes con ventilación mecánica prolongada.

3. Procedimientos urgentes:

a) Traqueostomía en pacientes con hipoxemia o hipercapnia como complicación de la colocación de traqueostomía reciente.

\section{Recomendaciones respecto a procedimientos pulmonares}

Si bien la broncoscopia es una estrategia útil en el diagnóstico de muchos procesos infecciosos pulmonares, dado el alto nivel de contagio, y el riesgo para el personal de salud, se considera como un procedimiento parcialmente CONTRAINDICADO en paciente con COVID y solo debe ser considerado en los siguientes casos:

- Pacientes intubados con pruebas de vías respiratorias altas negativas.

- Si existe la posibilidad de un diagnóstico alternativo concurrente que modifique el tratamiento.

- Condiciones que pongan en peligro la vida: obstrucción de la vía aérea, hemoptisis masivas, atelectasias con hipoxemia.

- El esputo inducido está contraindicado en la pandemia por COVID-19.

Se recomienda seguir las siguientes acciones por la seguridad de nuestros pacientes, equipo de trabajo y nuestra comunidad. Se prefiere la anestesia general e intubación endotraqueal.

\section{A. Protección del personal}

- Todo el personal de broncoscopia/sala de quirófano debe portar el equipo de protección individual (EPI) completo: mascarilla N95 o FPP3 y poner por encima otra mascarilla quirúrgica. Bata quirúrgica plastificada con manga larga. Gorro. Cobertor de calzado (calzas). Si es posible, se recomienda ponerse por encima un traje cerrado completo tipo "buzo" por encima de la bata. Siempre al final y deben ponerse la 
protección ocular con gafa cerrada tipo "buceador" (no valen las gafas "anti salpicaduras" abiertas lateralmente. Mantener la distancia entre el personal y los pacientes de 2 metros.

- El personal debe lavarse las manos con agua y jabón o alcohol gel antes y después del contacto con el paciente, así como previo y posterior a algún procedimiento.

- Limitar el personal que esté en contacto con el paciente (de preferencia dos).

- Limitar el tiempo de los procedimientos.

- El procedimiento debe ser realizado por el personal más experto. No puede haber personal en formación.

- En lo posible realizar COVID-19 test antes de realizar procedimiento.

- Los pacientes deben portar mascarilla de uso quirúrgico en el proceso preoperatorio y en el área de recuperación.

- Evitar realizar tratamiento con nebulizaciones para minimizar el riesgo de aerosoles.

\section{B. Broncoscopia para pacientes con bajo riesgo de infección por COVID-19}

- Bajo riesgo se define como:

a) 2 pruebas negativas para COVID-19.

b) Ausencia de fiebre, malestar general, síntomas respiratorios y que no haya viajado recientemente o haya estado expuesto con un caso confirmado de COVID-19.

- Todo el personal debe lavarse las manos con agua y jabón o alcohol gel como rutina y después de entrar a una sala de procedimientos.

- Equipo de protección: gorro, guantes, mascarillas quirúrgicas con careta facial, goggles, traje quirúrgico y protector de calzado.

- Seguir las recomendaciones CDC para la colocación y retiro adecuado del equipo de protección personal.

- Salas de procedimiento deben ser desinfectadas de manera estandarizada con un protocolo estricto.

- Seguir la estandarización de desinfección de los monitores reusables.

- Seguir la estandarización de desinfección de broncoscopios reutilizables.

a) El personal debe portar: gorro, mascarilla N95 con protector facial, traje quirúrgico y cobertores de calzado durante el procedimiento.

\section{Broncoscopia para pacientes con sospecha, enfermedad confirmada o no conocida por COVID-19}

- Aplicar todas las recomendaciones para pacientes de bajo riesgo de infección por $\mathrm{CO}$ VID-19 sumado a las siguientes.

- Debe evitar o retrasar el procedimiento a menos que la condición ponga en riesgo la vida del paciente.

- Todo el personal debe utilizar el equipo de protección personal.

a) Gorro desechable, mascarilla facial, traje quirúrgico desechable, mascarilla N95 con mascarilla facial, goggles, guantes y cobertor de calzado desechable.

- Se sugiere seguir las instrucciones del CDC para colocación y retiro del equipo de protección personal.

- Los procedimientos deben realizarse en un cuarto con presión negativa o en sala de cuidados críticos con filtro para partículas de alta eficiencia.

- Si se realiza un procedimiento donde exista aerosolización, fuera de la sala de procedimientos, es necesario notificar la necesidad de un filtro para partículas de alta eficiencia para control de infección.

- La broncoscopia rígida, si es necesaria, se hará en una sala con ventilación cerrada. Si es posible, se realizarán todos los procedimientos con un tubo orotraqueal. La intubación orotraqueal debe realizarse de manera ideal en terapia intensiva, previo a que se traslade al paciente a la sala de procedimientos.

- Evitar el uso de cualquier medicamento nebulizado por el riesgo de aerosoles.

- Una vez que el paciente ha dejado la sala de procedimientos, esta debe permanecer vacía por $30 \mathrm{~min}$ y posteriormente el equipo de trabajo puede entrar con mascarilla.

- Seguir la estandarización de desinfección de broncoscopios reusables.

a) El personal debe portar: gorro, mascarilla N95 con protector facial, goggles, traje quirúrgico y cobertores de calzado durante el procedimiento.

b) Si es posible, se recomienda ponerse por encima un traje cerrado completo tipo "buzo" por encima de la bata. Al finalizar el procedimiento o el paciente es extubado, debe utilizar mascarilla quirúrgica. Si 
requiere oxígeno, se debe administrar con la mascarilla quirúrgica puesta.

\section{Equipo de protección personal y selección de sala}

- Todos los pacientes deben cumplir con las precauciones de partículas modificadas excepto procedimientos que generen aerosoles.

- Uso de N95 o FPP3 para mediano y alto riesgo.

I. Procedimientos que generan alto riesgo de contagio:

- Inducción de esputo.

- Broncoscopia.

- Intubación y extubación traqueal.

- Aspiración abierta de tráquea.

- Uso de ventilación mecánica no invasiva u oxigeno de alto flujo.

- Colocación de sonda nasogástrica.

II. Procedimientos que general aerosol de mediano riesgo:

- Nebulizador (solo si el paciente tuvo tos persistente).

- Colocación de sonda nasogástrica.

- Cerrar la sala de procedimientos después del procedimiento, solo si el paciente previo tuvo tos persistente.

- Presión negativa en la sala de procedimiento durante 1 hora.

- Presión negativa en la sala general durante $2 \mathrm{~h}$.

III. Procedimientos que generan aerosoles de riesgo bajo:

- Colección de muestras nasofaríngeas.

- Ventilación mecánica no invasiva, con fugas menores a $10 \mathrm{lts} / \mathrm{min}$ y uso de filtro para virus.

- Uso de mascarilla quirúrgica y protección de ojos durante el procedimiento, NO es necesario cerrar la sala de procedimientos después de este.

\section{Procedimientos que no generan aerosoles:}

- Procedimientos que no se mencionan en la parte superior, incluyendo muestras orofaríngeas no son generadoras de aerosoles.

$V$. Selección de sala (presión negativa vs sala regular):

- Si está disponible, se prefiere presión negativa en el siguiente orden:
1. Necesidad de aislamiento por dispersión de partículas (ie: sospecha de tuberculosis).

2. Planeamiento de procedimientos que generan aerosoles.

3. Uso de ventilación mecánica no invasiva u oxigeno de alto flujo.

\section{E. Procedimientos pleurales}

I. Paciente con bajo riesgo para COVID-19:

- El procedimiento debe realizarse al lado de la cama o en sala de procedimientos.

- Pacientes deben tener un tamizaje con $24 \mathrm{~h}$ previas.

- El equipo de ultrasonido de tórax debe ser limpiado pre y post procedimiento con toallas desechables de peróxido de hidrógeno.

- Lavarse las manos con agua y jabón o alcohol gel, antes y posterior de entrar a servicio clínico o estar en contacto con pacientes.

- El personal debe limitarse a 2 personas durante el procedimiento.

- Equipo de protección personal previo a iniciar el procedimiento incluyendo guantes estériles, traje quirúrgico estéril, mascarilla facial, goggles y gorro desechable.

II. Paciente con COVID-19 positivo o sospechoso:

- El procedimiento debe realizarse al lado de la cama o en sala de procedimientos.

- Evaluar la urgencia del procedimiento.

- El personal debe limitarse a 2 personas durante el procedimiento.

- Si el paciente no está intubado, debe utilizar mascarilla quirúrgica y debe colocársela antes de entrar a la sala de procedimiento.

- Durante la estancia en la sala de procedimientos y durante el procedimiento pleural el equipo de trabajo debe de utilizar su equipo de protección personal:

1. Gorro quirúrgico.

2. Traje desechable.

3. Mascarilla N95 o FPP3.

4. Mascarilla facial con goggles.

5. Guantes.

6. Protector de calzado.

- El procedimiento debe suspenderse si se generan aerosoles en exceso.

- Seguir las instrucciones del CDC para colocación y retiro del equipo de protección personal. 


\section{F. Procedimientos misceláneos}

I. Traqueostomía percutánea (limitarse a dos personas por procedimiento):

- Determinar la urgencia del procedimiento y aplazarlo lo más posible.

- La traqueostomía debe ocurrir si los parámetros de ventilación mecánica se encuentren dentro de rango aceptable $\left(\left(\mathrm{FiO}_{2} \leq 0,6\right.\right.$ y PEEP $\leq 10)$.

- Se puede considerar una traqueostomía temprana con el fin de facilitar el traslado del paciente a una unidad de cuidado no crítico (si existe necesidad urgente de cama) o facilitar las medidas de higiene pulmonar.

- El procedimiento debe ser realizado en el cuarto de la terapia intensiva y con ello evitar la necesidad de traslado.

- Se debe elegir el tipo de técnica (percutánea versus quirúrgica) que menor tiempo lleve en realizarse (esto va a depender del expertise de cada centro).

- Con el fin de minimizar el riesgo de exposición; solo 2 miembros del personal deben estar presentes.

- Se seguirán los protocolos especificados previamente en el apartado "Broncoscopia para pacientes con sospecha, enfermedad confirmada o no conocida por COVID-19.

- Equipo de protección personal:

- Gorro quirúrgico.

- Traje desechable.

- PAPR (Powered Air Purified Respirator) y mascarilla N95 (preferiblemente) o mascarilla N95 y goggles.

- Guantes.

- Protector de calzado.

II. Gastrostomía percutánea (limitarse a 2 personas por procedimiento):

- Se recomienda aplazar en lo posible en pacientes con sospecha o confirmados de tener COVID 19 para minimizar el riesgo de contagio.

- Todos los pacientes hospitalizados deben tener un tamizaje de acuerdo con su historia clínica y ser discutido con equipo clínico para determinar la urgencia del procedimiento.

- Equipo de protección personal:

- Gorro quirúrgico.

- Traje desechable.
- Mascarilla N95 o PAPR.

- Mascarilla facial.

- Guantes.

- Protector de calzado.

III. Broncoscopia en el paciente sometido a oxigenación por membrana extracorpórea (ECMO) en caso de COVID-19:

- La evidencia actual es limitada y consiste principalmente en opinión de expertos.

- Las broncoscopias en esta población deben ser limitadas a aquellas consideradas como urgentes y que representan un riesgo inminente o para optimizar condiciones respiratorias y/o hemodinámicas.

- Equipo de protección personal: Gorro quirúrgico, traje desechable, PAPR (Powered Air Purified Respirator) y mascarilla N95 o mascarilla N95 y googles, guantes, protector de calzado.

- El personal dentro de la sala debe ser limitado al menor número posible.

- El procedimiento debe ser realizado a la cabecera del paciente por un neumólogo intervencionista minimizando la duración del mismo.

- Se seguirán los protocolos especificados previamente en el apartado "broncoscopia para pacientes con sospecha, enfermedad confirmada o no conocida por COVID-19".

\section{Recomendación sobre preguntas de tamizaje}

- Todos los pacientes que acudan a este servicio clínico o a sala de procedimientos se les llamará por teléfono $24 \mathrm{~h}$ previas a su cita programada: - ¿Ha tenido alguno de los siguientes síntomas?

i. Fiebre $\geq 38^{\circ} \mathrm{C}$.

ii. Escalofrío.

iii. Tos (basal o ha aumentado).

iv. Disnea (basal o ha aumentado).

v. Dolor de garganta.

vi. Pérdida del sentido del olfato y/o gusto. vii. Diarrea.

a. ¿En los últimos 14 días ha viajado fuera del país?

b. ¿En los últimos 14 días ha tenido contacto con una persona diagnosticada con $\mathrm{CO}$ VID-19? 
Tabla 1. Resumen de prioridad en procedimientos broncoscópicos

\section{Electivo}

Dilatación de estenosis traqueal o bronquial leve

Broncoscopia para drenaje de secreciones pulmonares

Sospecha de sarcoidosis sin indicación inmediata para tratamiento

Procedimientos diagnósticos para enfermedad intersticial pulmonar

Lavado bronco-alveolar para infecciones por el complejo Mycobacterium avium

Evaluación de tos crónica

Sospecha de enfermedad pulmonar infecciosa en el paciente inmunocomprometido

Broncoscopia dinámica para evaluación de traqueobroncomalacia y colapso dinámico excesivo de la vía aérea

Reducción de volumen pulmonar broncoscópica mediante válvulas endobronquiales

Termoplastía bronquial

Rheoplastía bronchial

Denervación pulmonar total

Instilación de Mitomycina-C o inyección de esteroide endobronquial

Resección de tumor endobronquial que NO ocasiona compromiso respiratorio

Hemoptisis leve o moderada

\section{Coagulación con argón plasma}

Resección de tumor endobronquial que ocasiona compromiso respiratorio

\section{Prioritario}

Biopsia de masa pulmonar o nódulo pulmonar con alta sospecha de cáncer

Biopsia de linfadenopatía hiliar o de mediastino sospechosa de cáncer

Lavado pulmonar total para tratamiento Hemoptisis masiva de la proteinosis alveolar primaria

Extracción de cuerpo extraño

Migración en la vía aérea de un stent

\section{Urgente}

Dilatación de estenosis traqueal o bronquial severa

Obstrucción sintomática de la vía área central 


\section{Referencias}

1. Group of Interventional Respiratory Medicine, Chinese Thoracic Society. [Expert consensus for bronchoscopy during the epidemic of 2019 novel coronavirus infection (Trial version)]. Zhonghua Jie $\mathrm{He} \mathrm{He} \mathrm{Hu} \mathrm{Xi} \mathrm{Za}$ Zhi. 2020 Mar 12;43(3):199-202. doi: 10.3760/cma.j.is sn.1001-0939.2020.03.012.

2. Repici A, Maselli R, Colombo M, Gabbiadini R, Spadaccini M, Anderloni A, Carrara S. Coronavirus (COVID-19) outbreak: what the department of endoscopy should know. Gastrointest Endosc. 2020 Mar 14. pii: S0016-5107(20)30245-5. doi:10.1016/j.gie.2020.03.019.
3. Wahidi MM, Lamb C, Murgu S, Musani A, Shojaee S, Sachdeva A, et al. American Association for Bronchology and Interventional Pulmonology (AABIP) Statement on the Use of Bronchoscopy and Respiratory Specimen Collection in Patients with Suspected or Confirmed COVID-19 Infection. J Bronchology Interv Pulmonol. 2020 Mar 18. doi: 10.1097/LBR.0000000000000681.

4. Pritchett MA, Oberg C, Belanger A, De Cardenas J, Cheng G, Cumbo-Nacheli G, et al. Society for Advanced Bronchoscopy Consensus Statement and Guidelines for Bronchoscopy and Airway Management Amid the COVID-19 Pandemic. Journal of Thoracic Diseases 2020 (accepted for publication). 